Eval uat i on techni que of ki net i c paramet er s for i rreversi bl e charge transfer reacti ons from st eady- st at e vol t ammogr ans at microdi sk el ect rodes

\begin{tabular}{|l|l|}
\hline 著者 & AOK Koi chi \\
\hline $\begin{array}{l}\text { j our nal or } \\
\text { publ i cat i on t i t l e }\end{array}$ & El ect rochemi st ry Communi cat i ons \\
\hline vol une & 7 \\
\hline nunber & 5 \\
\hline page r ange & $523-527$ \\
\hline year & $2005-05$ \\
\hline URL & ht t p: //hdl . handl e. net /10098/1148 \\
\hline
\end{tabular}




\title{
Evaluation technique of kinetic parameters for irreversible charge transfer reactions from steady-state voltammograms at microdisk electrodes
}

\author{
Koichi Aoki * \\ Department of Applied Physics, University of Fukui, 3-9-1 Bunkyo, Fukui-shi, 910-8507 Japan
}

\begin{abstract}
An explicit approximate expression for half-wave potentials of irreversible steady-state current-voltage curves at a microdisk electrode was derived from the diffusion equation and the Butler-Volmer equation on the basis of the previous analytical method (J. E. C., 235 (1987) 87). The plot of the half-wave potential, $E_{1 / 2}$, against $\left.\ln \left(1-\exp \left(-\left(E_{1 / 2^{-}} E^{0}\right)\right) F / R T\right)\right]$ showed a linear relation with the slope of $R T / \alpha F$, irrespective of the values of the kinetic parameters, where $a$ is the radius of the electrode and $\alpha$ is the transfer coefficient. Values on the both axis in this plot are readily accessible from the voltammograms. The intercept gave the rate constant. Values of kinetic parameters obtained from the disk model were very close to those from the hemisphere model although there is big difference in current distribution on the two model electrodes.
\end{abstract}

Keywords: microdisk electrode, electrode kinetics, hemispherical electrode, transfer rate constant, steady-state current, half-wave potential

\footnotetext{
* Corresponding author. Tel.: +81-776-278665; fax: + 81-90-8095-1906

E-mail address: d930099@icpc00.icpc.fukui-u.ac.jp (K. Aoki).
} 


\section{Introduction}

Nanometer-sized electrodes have revealed the behavior different from that observed at the conventional electrodes, exemplified by the deviation of the limiting current without deliberately adding supporting electrolyte at electrodes less than $10 \mathrm{~nm}$ from the theoretical values for the migration current $[1,2]$, dependence of estimated values of stability constants of metal complexes on the electrode radii [3], and the apparently large value, $220 \mathrm{~cm} \mathrm{~s}^{-1}$ [4], of the charge transfer rate constant of ferrocene estimated at the electrode $1.6 \mathrm{~nm}$ in radius in comparison with the value evaluated at a conventional microelectrode, $3.7 \mathrm{~cm} \mathrm{~s}^{-1}$ [5]. The last example has been examined by varying electrode radii [6]. The rate constant of hexacyanoferrate has apparently increased with a decrease in electrode radii both for the oxidation wave and the reduction one [7]. This increase has been explained in terms of non-negligible length of the mean free path of the diffusing redox particle in comparison with the electrode size [7]. It is still under debate whether the enhancement of rate constant is due to an intrinsic significance at a molecular level or an apparent overestimation by complicated mass transport [8].

These measurements have relied on the fabrication techniques of nanometer-sized electrodes, in which a nano-scaled hole is left behind from coating the electrode surface [9- 16]. Unfortunately, these electrodes bear some controversial problems about geometry of the exposed surface, more than one exposed domains, surface roughness, and cracks between the electrode and insulators [17-23]. In spite of the problems, these electrodes have been assumed to be either a disk or a hemisphere in geometry as a model for analyzing values of limiting currents and half-wave potentials.

Although the disk model is closer in geometry to real electrodes than the hemisphere model, the hemisphere model has been used for the kinetic analysis of a relation between the half-wave 
potential with the radii of the electrodes. This contradiction is obviously due to a less availability of simple theoretical equations at the disk model. Steady-state current-voltage curves at the disk electrode have been obtained rigorously by use of the Mellin transformation [24] and of the Bessel integration [25] for irreversible charge transfer reactions. However, a relation between the half-wave potential with the radii of the electrodes, which is practically useful, has not been given explicitly. This report presents a practically useful equation for the half-wave potential when a redox reaction is quasi-reversible and totally irreversible at the disk electrode.

\section{Evaluation of half-wave potential}

We concentrate on the one-electron slow redox reaction of soluble species at the disk electrode with the radius, $a$, under the steady-state condition. The equation for the current-voltage curves has been derived [24] on the assumptions that mass transport is controlled by diffusion with a diffusion coefficient, $D$, common to the oxidized and the reduced species, that the charge transfer kinetics is expressed by the Butler-Volmer equation, and that there is the reduced species with concentration, $c^{*}$, in the bulk. The current, $I$, at any potential, $E$, has been expressed as [24]

$$
I / I_{\mathrm{L}}=(2 / \sqrt{\pi})\left(1+\mathrm{e}^{-\varsigma}\right)^{-1} h_{1}
$$

where $\left(2 \pi^{-1 / 2}\right) h_{1}$ is the same as $f_{1}$ in the previous paper [24], and $\zeta$ is the dimensionless electrode potential, given by

$$
\varsigma=\left(E-E^{\mathrm{o}}\right) F / R T
$$

with the electrode potential, $E$, and the formal potential, $E^{\mathrm{o}} . I_{\mathrm{L}}$ is the diffusion-controlled limiting current given by

$$
I_{\mathrm{L}}=4 F C * D a
$$


Function $h_{k}$ for integer $k(=1,2,3, \ldots)$ has been given by

$2 h_{k}+\frac{4(2 k-1)}{\pi \lambda} \sum_{m=1}^{\infty} \frac{h_{m}}{(2 m-2 k+1)}=\sqrt{\pi}$

Here, $\lambda$ is the kinetic parameter, defined as

$\lambda=(a / D)(\vec{k}+\overleftarrow{k})=\left(k^{\mathrm{o}} a / D\right) \mathrm{e}^{\alpha \varsigma}\left(1+\mathrm{e}^{-\varsigma}\right)$

This includes the forward (anodic) rate constant, $\vec{k}$, the backward (cathodic) rate constant, $\overleftarrow{k}$, the standard rate constant, $k^{\circ}$, and the anodic transfer coefficient, $\alpha$.

The half-wave potential, $E_{1 / 2}$ or $\zeta_{\mathrm{h}}=\left(E_{1 / 2}-E^{0}\right) F / R T$, is defined by the potential at which $I$ $=I_{\mathrm{L}} / 2$. Eq.(1) gives the relation between $\zeta_{\mathrm{h}}$ and $h_{1}$ as

$$
\varsigma_{\mathrm{h}}=-\ln \left(4 h_{1} / \sqrt{\pi}-1\right)
$$

Our aim here is to obtain the dependence of $E_{1 / 2}$ on $\ln (a)$, which corresponds to the variation of $\zeta_{\mathrm{h}}$ with $\lambda$ through $h_{1}$. Values of $h_{\mathrm{k}}$ can be obtained by replacing $\infty$ in Eq.(4) by a large integer, $M$, and by taking $k$ to be from 1 to $M$.

Values of $h_{1}$ thus computed are plotted against $\log (\lambda)$ in Fig. 1 . The condition, $\mathrm{e}^{-\zeta}<<1$, represents the totally irreversible reaction because $\mathrm{e}^{-\zeta}$ and 1 in Eq.(5) result from the backward and the forward reaction rate constant, respectively. Equation (5) to which this condition is applied becomes

$$
\ln \lambda_{\mathrm{ir}}=\ln \left(k^{\mathrm{o} a} / D\right)+\alpha\left(E-E^{\mathrm{o}}\right) F / R T
$$

This represents the dimensionless potential. In contrast, Eq.(1) for $\mathrm{e}^{-\zeta}<<1$ indicates that $\left(2 \pi^{-1 / 2}\right) h_{1}$ gives the normalized current. Therefore, the curve in Fig.1 expresses the current-voltage curve for the totally irreversible reaction. When current-voltage curves or half-wave potentials are analyzed, it would be convenient to have a simple approximate equation. We obtained the approximate equation in the following form

$$
I / I_{\mathrm{L}}=\left(1+\mathrm{e}^{-\varsigma}\right)^{-1} \lambda /(1.38+\lambda)
$$

Values of $\lambda /(1.38+\lambda)$ are plotted in Fig. 1 as circles. The maximum errors are $1.9 \%$ at $\log (\lambda)=1$. 
A technique of deriving Eq.(8) will be described in the later section.

\section{Results and Discussion}

We obtained a relationship between the dimensionless half-wave potential, $\zeta_{\mathrm{h}}$, and the kinetic parameter, $a k^{\mathrm{o}} / D$, for a given value of $\alpha$, according to the following process: $h_{1}$ was evaluated for a given value of $\lambda$ from Eq.(4); it yielded a value of $\zeta_{\mathrm{h}}$ from Eq.(6); a value of $a k^{\mathrm{o}} / D$ was obtained from Eq.(5) for known values of $\lambda, \alpha$ and $\zeta_{\mathrm{h}}$. Values of the half-wave potential thus obtained were plotted against $a k^{\mathrm{o}} / D$ in Fig.2 for several values of $\alpha$. With a decrease in the electrode size, the steady-state anodic voltammograms shift in the positive direction. If the potential shift more than $5 \mathrm{mV}$ from $E^{\mathrm{o}}\left(\zeta_{\mathrm{h}}>0.19\right)$ is regarded as the irreversible system, the diagnosis of the irreversiblity is given by $\ln \left(a k^{\mathrm{o}} / D\right)<2.3$ or $a k^{0}<10^{-4} \mathrm{~cm}^{2} \mathrm{~s}^{-1}$ for $D$ $=10^{-5} \mathrm{~cm}^{2} \mathrm{~s}^{-1}$ (see Fig.2). If voltammograms at electrodes with $a=1 \mu \mathrm{m}$ and $10 \mathrm{~nm}$ show $5 \mathrm{mV}$ potential shift, the reaction rate constants are $k^{0}=1$ and $100 \mathrm{~cm} \mathrm{~s}^{-1}$, respectively. On the other hand, the variation of $\zeta_{\mathrm{h}}$ with $\ln \left(a k^{\mathrm{o}} / D\right)$ tends to a straight line with a slope $1 / \alpha$ with a decrease in $\ln \left(a k^{\mathrm{o}} / D\right)$, (shown as dashed line (f) in Fig.2). The region of $\ln \left(a k^{\mathrm{o}} / D\right)<-0.8$ or $a k^{\mathrm{o}}<4 \times 10^{-6}$ $\mathrm{cm}^{2} \mathrm{~s}^{-1}$ for $D=10^{-5} \mathrm{~cm}^{2} \mathrm{~s}^{-1}$ is the totally irreversible domain.

It would be interesting to compare the curves in Fig. 2 with those at a hemispherical electrode with radius $a$. The concentrations of the oxidized and the reduced species at the electrode surface under the steady-state are expressed, respectively, by

$$
\left(c_{\mathrm{O}}\right)_{r=a}=-(a / D)\left(I / 2 \pi a^{2} F\right) \quad\left(c_{\mathrm{R}}\right)_{r=a}=c^{*}-(a / D)\left(I / 2 \pi a^{2} F\right)
$$

When both equations are inserted into the Butler-Volmer equation, the current can be expressed by

$$
I=\frac{2 \pi a^{2} F C * \vec{k}}{1+(a / D)(\vec{k}+\overleftarrow{k})}
$$

When this is divided by the diffusion-controlled limiting current at the hemispherical electrode 
$I_{\mathrm{L}}=2 \pi a F C * D$

we obtain the expression for the normalized current

$$
I / I_{\mathrm{L}}=\vec{k} a / D(1+\lambda)=\lambda(1+\lambda)^{-1}\left(1+\mathrm{e}^{-\varsigma}\right)^{-1}
$$

Taking $I / I_{\mathrm{L}}=1 / 2$ yields the normalized half-wave potential at the hemispherical electrode, expressed by

$$
\exp \left(\alpha \varsigma_{\mathrm{h}}\right)\left[1-\exp \left(-\varsigma_{\mathrm{h}}\right)\right]=D / k^{\mathrm{o}} a
$$

The variation of $\zeta_{\mathrm{h}}$ with $\ln \left(a k^{\mathrm{O}} / D\right)$ calculated from Eq.(12) for $\alpha=0.5$ is shown in Fig.2 as a dashed curve. The curve shifted by 0.32 along the $\ln \left(a k^{\mathrm{O}} / D\right)$-axis is almost overlapped with curve (c) for the disk electrode at $\alpha=0.5$. The shift is equivalent to the replacement of $a$ by $0.73 a\left(\mathrm{e}^{-0.32}=0.73=1 / 1.38\right)$. In other word, the disk electrode is 1.38 times more efficient in size to the electrode kinetics than the hemispherical electrode. The efficiency is obviously caused by the high current density at the edge.

When the radius of the hemispherical electrode in Eq.(11) was replaced by $0.73 a$ of the disk electrode, Eq.(8) was obtained. Applying this technique to Eq.(12), we obtain the approximate equation for the half-wave potential at the disk electrode

$$
\exp \left(\alpha \varsigma_{\mathrm{h}}\right)\left[1-\exp \left(-\varsigma_{\mathrm{h}}\right)\right]=1.38 D / k^{0} a
$$

This equation contains maximum errors of $1.9 \%$ in the quasi-reversible domain.

We compare values of $k^{0} /$ at the disk electrode with those at the hemisphere electrode when a voltammogram shows $I_{\mathrm{L}}=10 \mathrm{pA}$ and $E_{1 / 2}-E^{0}=0.1 \mathrm{~V}$ under the conditions of $c^{*}=2.5$ $\mathrm{mM}$ and $D=10^{-5} \mathrm{~cm}^{2} \mathrm{~s}^{-1}$. The radius can be estimated from the diffusion-controlled limiting current by use of Eq.(3) or (10), and we obtain $(a)_{\text {disk }}=10 \mathrm{~nm}$ and $(a)_{\text {sphere }}=6.4 \mathrm{~nm}$. Applying $E_{1 / 2}-E^{\mathrm{o}}=0.1 \mathrm{~V}$ to the curves for $\alpha=0.5$ in Fig.2, we get $\ln \left(a k^{\mathrm{o}} / D\right)_{\text {disk }}=-1.60$ and $\ln \left(a k^{\mathrm{o}} / D\right)_{\text {sphere }}$ $=-1.92$, which correspond to $\left(k^{0}\right)_{\text {disk }}=2.0 \mathrm{~cm} \mathrm{~s}^{-1}$ and $\left(k^{0}\right)_{\text {sphere }}=2.3 \mathrm{~cm} \mathrm{~s}^{-1}$. The values of $k^{\mathrm{o}}$ are actually the same, indicating that either model is allowed to be used for evaluation of the rate constant. 
We consider a graphical technique of evaluating $\alpha$ and $k^{\circ}$. Experimentally known values in Eq.(13) are $E_{1 / 2}, E^{0}, D$ and $a$ under conventional conditions. We rewrite Eq.(13) to the following form:

$\alpha\left(E_{1 / 2}-E^{\mathrm{o}}\right) F / R T=0.32+\ln \left(D / k^{\mathrm{o}}\right)-\ln \left(a\left[1-\exp \left(-\varsigma_{\mathrm{h}}\right)\right]\right)$

When values of $E_{1 / 2}-E^{0}$ are plotted against values of $\ln \left(a\left[1-\exp \left(-\zeta_{\mathrm{h}}\right)\right]\right)$, the plots may fall on a straight line with the slope of $R T / \alpha F$. The extrapolation of the plot toward $E_{1 / 2}-E^{o}=0$ gives a value of $\ln \left(D / k^{0}\right)$ through $-0.32+\ln \left(a\left[1-\exp \left(-\zeta_{\mathrm{h}}\right)\right]\right)_{\text {intercept, }}$ where $\left(a\left[1-\exp \left(-\zeta_{\mathrm{h}}\right)\right]\right)_{\text {intercept }}$ is a value on the axis at the intercept rather than the value substituting $E_{1 / 2}-E^{0}=0$ Consequently, we can evaluate values of $k^{\circ}$ and $\alpha$ from this plot.

We have the data of the half-wave potentials of steady-state voltammograms for hexacyanoferrate (II) and (III) at various radii ranging from nanometer to micrometer [7]. The value of $E^{0}$ was determined by voltammograms at large electrode at slow scan rates. Values of $\zeta_{\mathrm{h}}$ for the oxidation of hexacyanoferrate (II) were plotted against $\ln \left(a\left[1-\exp \left(-\zeta_{\mathrm{h}}\right)\right]\right)$ as open circles in Fig.3, whereas those for the reduction of hexacyanoferrate (III) were plotted against $\ln \left(a\left[1-\exp \left(\zeta_{\mathrm{h}}\right)\right]\right)$ as fill circles. Both plots fell on each straight line if very small values of $\zeta_{\mathrm{h}}$ are neglected. From the slopes, we estimated the anodic and the cathodic transfer coefficient to be 1.2 and 2.1, respectively. These values are not only quite deviated from the conventional value, 0.5 , but also not satisfied with the condition of the unity of the sum of the anodic and the cathodic transfer coefficient. The point here is to fall on a straight line rather than the values of $\alpha$.

It is worth while noting that Eq.(14) is based on the simple combination of diffusion with the Butler-Volmer equation. It is not clear whether this simplification is valid for nanometer scaled electrodes. Possible reasons for the deviation are the assumption of continuous medium [7], the neglect of an inertia term for the law of motion in hydrodynamics, hydrodynamic interactions [26,27], delay of diffusion by collision of the oxidized species and the reduced 
species, a decrease in the activation energy at large flux [28], a decrease in hydrodynamic resistance against diffusing particles with high velocity $[29,30]$, and double layer effects on the kinetics [31]. However, these problems are beyond the subject of this report.

\section{Conclusion}

The graphical technique of evaluating the kinetic parameters from half-wave potential and the electrode radii was presented here by use of the previous theoretical analysis. A key plot is the linear relation of Eq.(14), in which the variables in both $x$ - and $y$-axis are voltammetrically available. The linearity is valid also for the quasi-reversible case. The slope yields the transfer coefficient, whereas the intercept gives transfer rate constant for a known value of the common diffusion coefficient.

The edge effect of the disk electrode, which makes the current be charge transfer controlled, can be represented as $16 \mathrm{mV}$ of $\left(E_{1 / 2}\right)_{\text {disk }}-\left(E_{1 / 2}\right)_{\text {sphere }}\left(=0.32\right.$ of $\ln \left(a k^{\mathrm{o}} / D\right)$ or $\left.\zeta_{\mathrm{h}}\right)$ at $\alpha=$ 0.5 when the disk electrode has the same radius as the hemispherical electrode. This difference in $E_{1 / 2}$ corresponds to $10 \%$ difference in the charge transfer rate constant. Although the disk electrode should show the current distribution quite different from the distribution at the hemispherical electrode, the difference in $k^{0}$ is small enough for kinetic data.

\section{Acknowledgement}

This work was financially supported by Grants-in-Aid for Scientific Research (Grants 14340232) from the Ministry of Education in Japan.

\section{References}

[1] J. L. Conyers Jr., H. S. White, Anal. Chem., 72 (2000) 4441.

[2] V. P. Menon, C. R. Martin, Anal. Chem., 67 (1995) 1920. 
[3] J. Galceran, J. Puy, J. Salvador, J. Cecilia, H. P. van Leeuwen, J. Electroanal. Chem., 505 (2001) 85 .

[4] R. M. Penner, M. J. Heben, T. L. Longin, N. S. Lewis, Science. 250 (1990) 1118.

[5] M. V. Mirkin, T. C. Richards, A. J. Bard, J. Phys. Chem., 97 (1993) 7672.

[6] J. J. Watkins, J. Chen, H. S. White, H. D. Abruna, E. Maisonhaute, C. Amatore, Anal. Chem., $75(2003) 3962$.

[7] J. Chen, K. Aoki, Electrochem. Commun., 4 (2002) 24.

[8] J. D. Norton, H. S. White, S. W. Feldberg, J. Phys. Chem., 94 (1990) 6772.

[9] R. Sonnenfeld, P. Hansma, Science, 232 (1986) 221.

[10] H. Liu, F.-R. Fan, C. W. Lin, A. J. Bard, J. Am. Chem. Soc., 108 (1986) 3838.

[11] D. J. Trevor, C. E. Chiclsey, D. N. Loiacono, Phys. Rev. Lett., 62 (1989) 929.

[12] A. A. Gewirth, D. H. Graston, A. J. Bard, J. Electroanal. Chem., 261 (1989) 477.

[13] M. P. Greer, K. J. Hanson, D. A. Scherson, X. Xing, M. Richter, P. N. Ross, R. Carr, I. Lindau, J. Chem. Phys., 93 (1989) 2181.

[14] C. M. Vitus, S.-C. Chang, B. C. Schardt, M. J. Weaver, J. Phys. Chem., 95 (1991) 7559.

[15] C. E. Bach, R. J. Nichols, W. Beckmann, H. Meyer, J. Electrochem. Soc., 140 (1993) 1281.

[16] C. J. Slevin, N. J. Gray, J. V. Macpherson, M. A. Webb, P. R. Unwin, Electrochem. Commun., 1 (1999) 282.

[17] R. A. Saraceno, A. G. Ewing, J. Electroanal. Chem., 257 (1988) 83.

[18] R. M. Penner, M. J. Heben, N. S. Lewis, Anal. Chem. 61 (1989) 1630.

[19] B. D. Pendley, H. D. Abruna, Anal. Chem. 62 (1990) 782.

[20] C. Lee, C. J. Miller, A. J. Bard, Anal. Chem., 63 (1991) 78.

[21] K. T. Kawagoe, J. A. Jankowski, R. M. Wightman, Anal. Chem., 63 (1991) 1589.

[22] T. G. Strein, A. G. Ewing, Anal. Chem., 64 (1992) 1368.

[23] M. V. Mirkin, F. -R. F. Fan, A. J. Bard, Science, 257 (1992) 364. 
[24] K. Aoki, K. Tokuda, H. Matsuda, J. Electroanal. Chem., 235 (1987) 87.

[25] K B. Oldham, C. G. Zoski, J. Electroanal. Chem., 313 (1991) 17.

[26] P. N. Pusey, R. J. A. Tough, Faraday Discuss. Chem. Soc., 76 (1983) 123.

[27] M. Doi, S. F. Edwards, The Theory of Polymer Dynamics, Clarendon Press, Oxford, 1986, pp. 65-68.

[28] K. Aoki, Review of Polarography, 50 (2004) 279.

[29] R. B. Bird, R. C. Armstrong, O. Hassager, Dynamics of Polymeric Liquids, Wiley, 1996, Vol. 1, Chapt. 1.

[30] I. Proudman, J. R. A. Pearson, J. Fluid Mech., 2 (1957) 237.

[31] R. B. Morris, D. J. Franta, H. S. White, J. Phys. Chem., 91 (1987) 3559. 
Figure Captions

Fig.1. Variation of $2 \pi^{-1 / 2} h_{1}$ with $\log (\lambda)$ calculated from Eq.(4) for $M=800$. Circles were evaluated from the approximation of Eq.(8).

Fig.2. Dependence of the dimensionless half-wave potential, $\zeta_{\mathrm{h}}$, on the dimensionless reaction rate constant, $a k^{\mathrm{o}} / D$, on logarithmic scale for $\alpha=$ (a) 0.3 , (b) 0.4 , (c) 0.5 , (d) 0.6 and (e) 0.7. Line (f) is the asymptotic line for curve (e). The dotted curve is for the hemispherical electrode for $\alpha=0.5$.

Fig.3 Plots of the dimensionless half-wave potential, $\zeta_{\mathrm{h}}$, for hexacyanoferrate against the logarithm of the modified radius, according to Eq.(14). The signs of $\zeta_{\mathrm{h}}$ on the abscissa are taken to be - and + for the anodic wave and the cathodic one, respectively. 


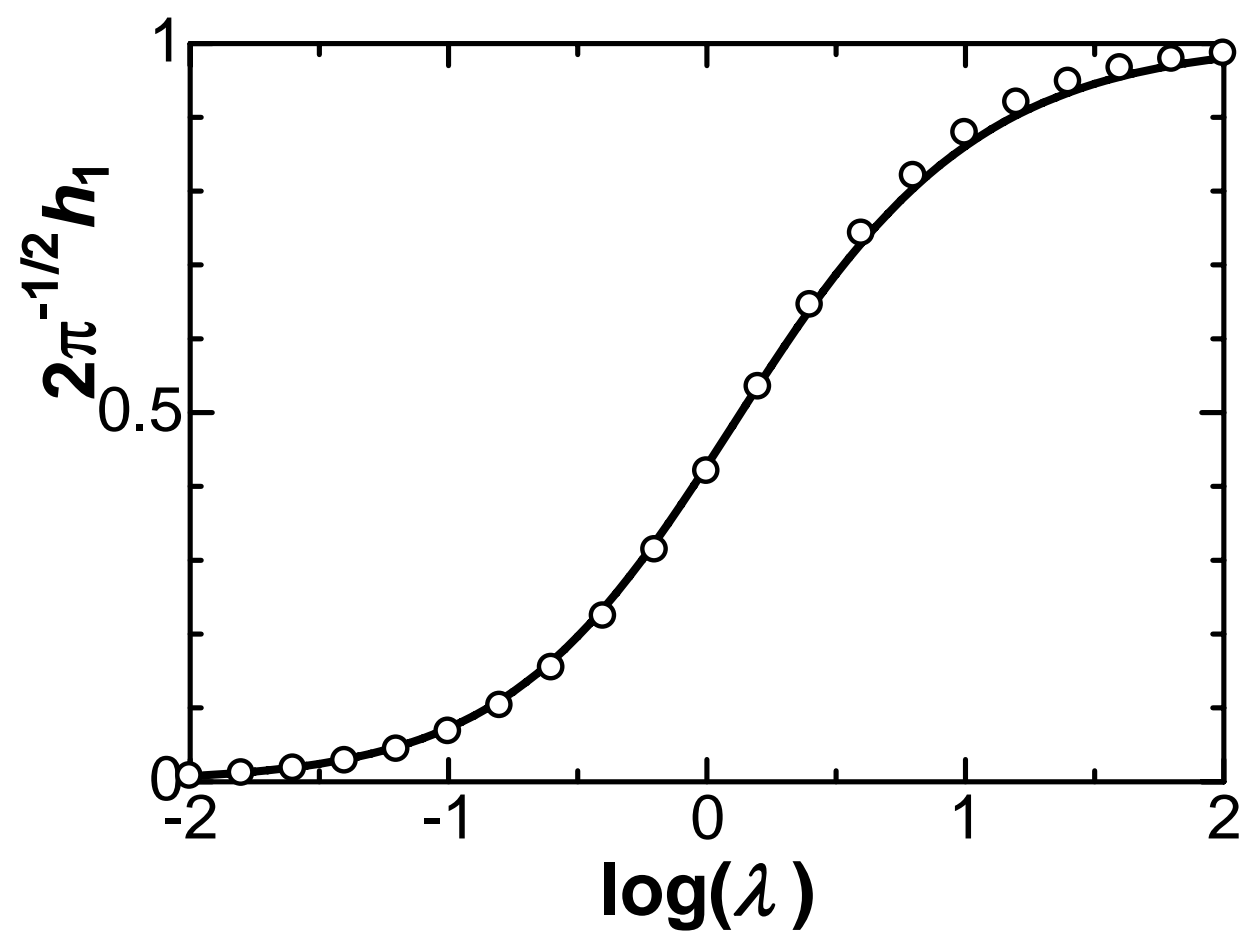

Fig.1. K. Aoki

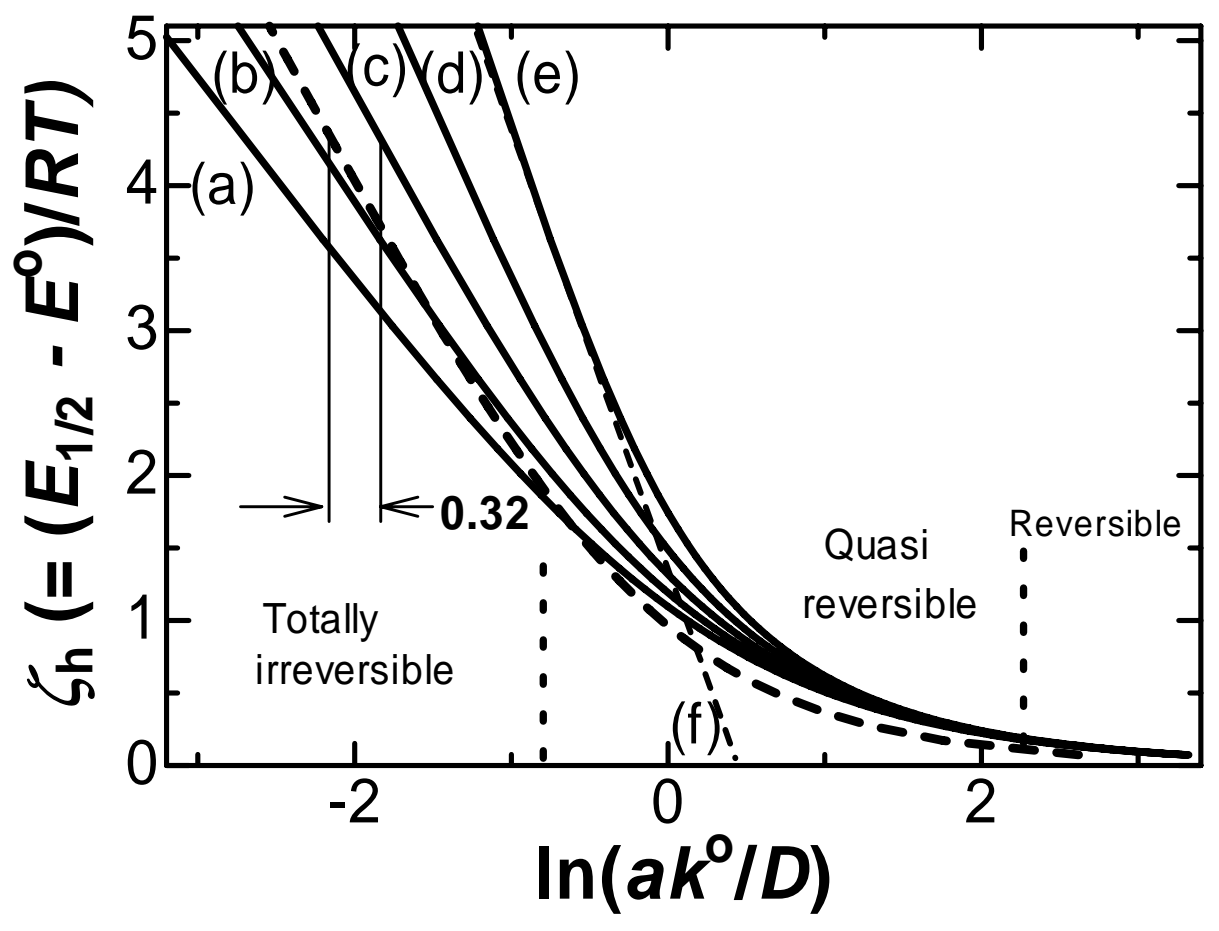

Fig. 2 K. Aoki 


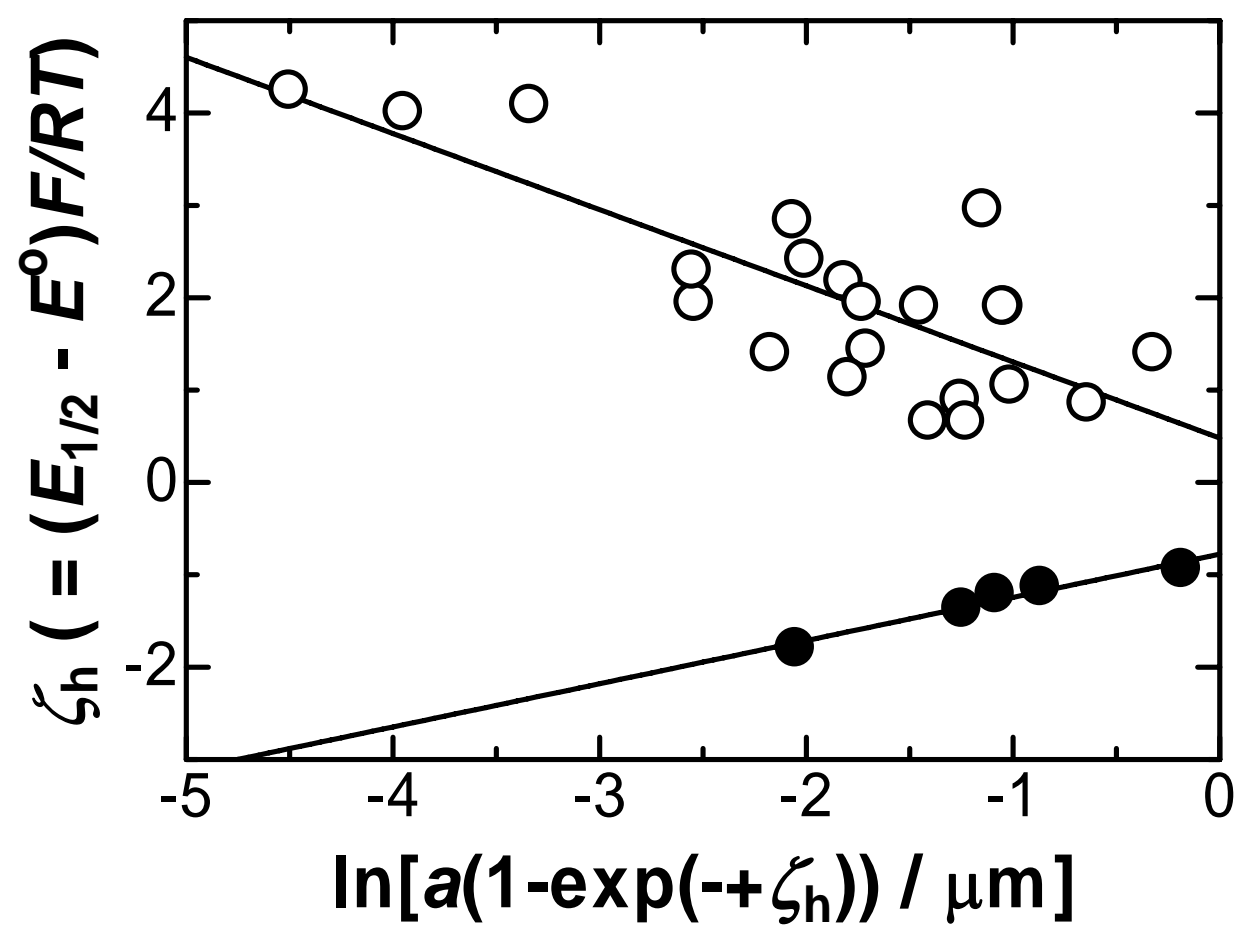

Fig.3 K. Aoki 